\title{
Guidelines for Use of the Approximate Beta-Poisson Dose-Response Model
}

Gang $\mathrm{Xie}^{1,2^{*}}$, Anne Roiko ${ }^{2,3}$, Helen Stratton ${ }^{2}$, Charles Lemckert ${ }^{2,4}$, Peter K. Dunn ${ }^{1}$,and Kerrie Mengersen ${ }^{5}$

${ }^{1}$ Faculty of Science, Health, Education and Engineering, University of the Sunshine Coast, Queensland, Australia.

${ }^{2}$ Smart Water Research Centre, Griffith University, Queensland, Australia.

${ }^{3}$ Menzies Health Institute Queensland, Griffith University, Queensland, Australia.

${ }^{4}$ Griffith School of Engineering, Griffith University, Queensland, Australia.

${ }^{5}$ Science and Engineering Faculty, Queensland University of Technology, Queensland, Australia.

*Address correspondence to Gang Xie, Building G51, Gold Coast Campus, Griffith University, Queensland 4222, Australia; g.xie@griffith.edu.au. 


\begin{abstract}
For dose-response analysis in Quantitative Microbial Risk Assessment (QMRA), the exact beta-Poisson model is a two-parameter mechanistic dose-response model with parameters $\alpha>0$ and $\beta>$ 0, which involves the Kummer confluent hypergeometric function. Evaluation of a hypergeometric function is a computational challenge. Denoting $P_{I}(d)$ as the probability of infection at a given mean
\end{abstract} dose $d$, the widely used dose-response model $P_{I}(d)=1-\left(1+\frac{d}{\beta}\right)^{-\alpha}$ is an approximate formula for the exact beta-Poisson model. Notwithstanding the required conditions $\alpha \ll \beta$ and $\beta \gg 1$, issues related to the validity and approximation accuracy of this approximate formula have remained largely ignored in practice, partly because these conditions are too general to provide clear guidance. Consequently, this study proposes a probability measure $\operatorname{Pr}(0<r<1 \mid \hat{\alpha}, \hat{\beta})$ as a validity measure ( $r$ is a random variable that follows a gamma distribution; $\hat{\alpha}$ and $\hat{\beta}$ are the maximum likelihood estimates of $\alpha$ and $\beta$ in the approximate model); and the constraint conditions $\hat{\beta}>(22 \hat{\alpha})^{0.50}$ for $0.02<\hat{\alpha}<2$ as a rule-of-thumb to ensure an accurate approximation (e.g., $\operatorname{Pr}(0<r<1 \mid \hat{\alpha}, \hat{\beta})>0.99)$. This validity measure and rule-ofthumb were validated by application to all the completed beta-Poisson models (related to 85 datasets) from the QMRA community portal (QMRA Wiki). The results showed that the higher the probability $\operatorname{Pr}(0<r<1 \mid \hat{\alpha}, \hat{\beta})$, the better the approximation. The results further showed that, among the total 85 models examined, 68 models were identified as valid approximate model applications which all had a near perfect match to the corresponding exact beta-Poisson model dose-response curve.

Key words: QMRA; beta-Poisson dose-response model; a rule-of-thumb; experimental dose-response data. 


\section{INTRODUCTION}

Quantitative Microbial Risk Assessment (QMRA) ${ }^{(1,2)}$ is a valuable framework for analysing the adverse health outcomes associated with exposures to pathogenic organisms. ${ }^{(3,4)}$ The QMRA approach is a fourstep process comprising (i) hazard identification; (ii) exposure assessment; (iii) dose-response analysis; and (iv) risk characterization. ${ }^{(1,2)}$ QMRA has become increasingly accepted by health authorities and is referenced in many major health guideline documents pertaining to water-borne pathogens (e.g., drinking water, ${ }^{(5,6)}$ wastewater irrigation, ${ }^{(7-9)}$ recreational waters, $\left.{ }^{(10)}\right)$ and to food-safety. ${ }^{(6,11)}$ QMRA has also been used widely by researchers to characterize microbial risks associated with food, water, and wastewater use in agriculture. ${ }^{(1,3,12)}$ The core part of the QMRA framework is the dose-response analysis which models the mathematical characterization of the relationship between the dose administered and the probability of adverse effect (typically, the probability of infection) in the exposed population. ${ }^{(1,2)}$ Among different microbial dose-response models proposed in the QMRA literature, the beta-Poisson model remains as the most popular plausible dose response model. ${ }^{(1,2,6)}$ The beta-Poisson dose-response model and its special case, the exponential dose-response model, have been well studied and widely employed to characterize infectivity of various viral, bacterial, and protozoan pathogens since the 1980s. ${ }^{(1,2,6,13,14)}$

Due to the mathematical complexity in its model specification and the difficulty in parameter estimation in the exact beta-Poisson model (model specification details are presented in Section 2.2), an approximate

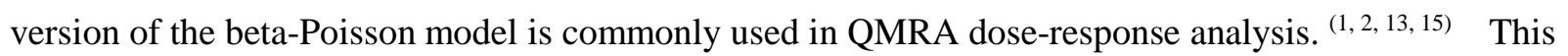
approximate beta-Poisson model is specified as

$$
P_{I}(d)=1-\left(1+\frac{d}{\beta}\right)^{-\alpha},
$$

where $d$ represents the known mean dose; $\alpha>0$ and $\beta>0$ are the model parameters to be estimated. ${ }^{(1,15 \text {, }}$

16) It is well known that the valid application of Equation (1) is subject to the general rule: $\alpha \ll \beta$ and $\beta \gg 1,^{(1,15,16)}$ but no commonly agreed, specific rules are available in the QMRA literature for researchers and practitioners to apply for the assessment of the validity or the approximation performance 
of the approximate beta-Poisson model (Equation (1)). Two things make these assessment conditions difficult to apply: (1) the true parameters, $\alpha$ and $\beta$ are unknown; (2) the general rule conditions are not specific enough to apply. As a consequence, the validity and approximation accuracy issue in the application of the approximate model has remained largely ignored. This study aims to address this issue. Based on theoretical justification, a gamma distribution probability measure is proposed as a validity measure upon which a rule-of-thumb is specified to ensure an accurate approximation in the applications of the approximate beta-Poisson dose-response model. Importantly, the rule-of-thumb uses the maximum likelihood estimates (MLEs) of $\alpha$ and $\beta$ ( $\hat{\alpha}$ and $\hat{\beta}$ ) obtained from the approximate model (i.e., Equation (1)). Therefore, this study provides revised guidelines which are practical to apply for the assessment of a valid use of the approximate beta-Poisson model.

The QMRA Wiki ${ }^{(2)}$ is a community portal for current quantitative information and knowledge developed for the quantitative microbial risk assessment field. This study has taken the advantage of having the free access to the possibly most complete collection of the experimental dose-response datasets reported in the literature as now available from the QMRA Wiki(2). The proposed rule-of-thumb is applied to all the completed beta-Poisson models (related to 85 datasets) presented in the QMRA Wiki ${ }^{(2)}$ for validation. The rule-of-thumb is validated through matching the dose-response curves between the approximate models and the corresponding exact models, and by taking into account the uncertainty in parameter estimation. The research results from this study are expected to provide a better understanding and facilitate the future applications of the beta-Poisson models in QMRA practice.

The rest of the paper is organized as follows. Section 2 provides a description of the theoretical background (model specifications and the parameter estimation procedure), and the data and notation used throughout the study. Section 3 provides the theoretical and empirical justification for developing the rule-of-thumb and describes the validation procedure for this rule-of-thumb. The validation results are then presented and discussed in Section 4, illustrating how the rule-of-thumb is validated through matching the dose-response curves and demonstrates how uncertainty is taken into account in this validation process using confidence bands. Section 5 presents the conclusions drawn from the research. 


\section{NOTATION, THEORETICAL BACKGROUND, AND DATA}

\subsection{Notation And Definitions}

For the purpose of a clear and consistent model specification and easy comparison with the literature results, we have adopted a notation scheme as detailed in Table I throughout this paper. The term 'organism' is used as a short name for pathogenic microorganism.

Table I. Notation and definition

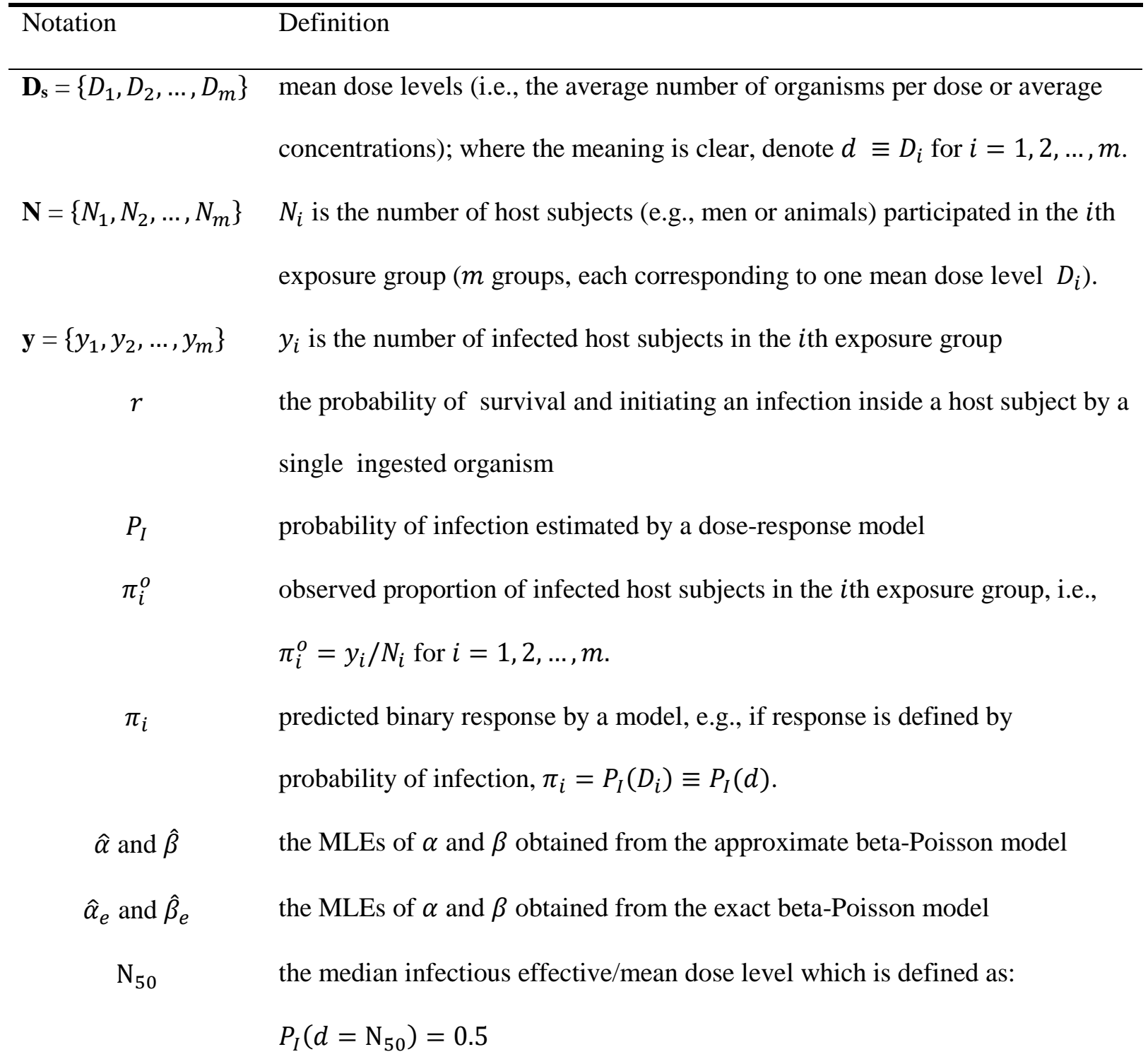


$\exp ($.$) and \log \quad \exp (1)=e \approx 2.71828$ is the natural logarithm base such that $\log (e)=1$.

$\Gamma(\cdot) \quad$ the gamma function ${ }^{(17)}$

\subsection{Model Specification}

The detailed description and derivation of the exponential and the beta-Poisson dose-response models can be found in the QMRA literature, e.g., Haas et al (2014) ${ }^{(1)}$. For the purpose of this paper, the following model specifications are needed.

Based on the assumptions that (i) the actual number of organisms ingested by a host subject (e.g., a human or an animal) is a random variable which follows a Poisson distribution with parameter $d$ (the known mean dose, i.e., the dose is assumed to have Poisson error ${ }^{(15,18)}$ ); (ii) one organism is capable of initiating an infection if it arrives at an appropriate site within a host subject; (iii) organisms have independent and identical probability $(r)$ of surviving to reach and initiate an infection at an appropriate site, the simplest QMRA dose-response model (the exponential model) can be derived as: ${ }^{(1,18)}$

$$
P_{I}(d)=1-\exp (-r d) .
$$

In Equation (2), if we take into account the host susceptibility or host sensitivity by allowing $r$ to follow a beta distribution with the probability density function (pdf):

$$
f(r \mid \alpha, \beta)=\frac{\Gamma(\alpha+\beta)}{\Gamma(\alpha) \Gamma(\beta)} r^{\alpha-1}(1-r)^{\beta-1},
$$

where $0<r<1$, and parameters $\alpha>0$ and $\beta>0$, we obtain the well-known exact beta-Poisson doseresponse model: ${ }^{(1)}$

$$
P_{I}(d)=1-{ }_{1} F_{1}(\alpha, \alpha+\beta,-d)
$$

where ${ }_{1} F_{1}(\alpha, \alpha+\beta,-d)$ is the Kummer confluent hypergeometric function ${ }^{(19)}$ defined by 


$$
{ }_{1} F_{1}(\alpha, \alpha+\beta,-d)=1+\frac{\Gamma(\alpha+\beta)}{\Gamma(\alpha)} \sum_{j=1}^{\infty}\left[\frac{\Gamma(\alpha+j)}{\Gamma(\alpha+\beta+j)} \frac{(-1)^{j-1}(d)^{j}}{j !}\right]
$$

Since there are no analytic solutions to ${ }_{1} F_{1}(\alpha, \alpha+\beta,-d)$, only approximate solutions or asymptotic solutions can be obtained ${ }^{(19,20)}$. In their original paper, Furumoto and Mickey ${ }^{(16)}$ derived the attractive approximation beta-Poisson dose-response formula: $P_{I}(d)=1-\left(1+\frac{d}{\beta}\right)^{-\alpha}$ (Equation (1)).

The approximate formula (Equation (1)) provided a simple solution for those using QMRA to circumvent the computational difficulties caused by the inherent mathematical complexity of the exact beta-Poisson dose-response model (Equation (4)). By series expansion, Furumoto and Mickey (1967) ${ }^{(16)}$ showed that, given a fixed value of $\alpha$, for sufficiently large values of $\beta$, Equation (1) is a valid approximation for Equation (4); they also asked readers to pay attention to the cases when $\alpha<1$ and $\beta>1$, and a particular example with $\alpha=0.0001$ and $\beta=4.0$ was given to show a good approximation of Equation (1) for Equation (4). Many years later, Teunis and Havelaar (2000) ${ }^{(15)}$ noted that Equation (1) can be derived from a different perspective. By starting again from the exponential model (Equation (2)), let variable $r$ follow a gamma distribution with the pdf:

$$
f(r \mid \alpha, \beta)=\frac{\beta^{\alpha}}{\Gamma(\alpha)} r^{\alpha-1} \exp (-\beta r)
$$

where $r>0$, and $\alpha>0$ and $\beta>0$ are the parameters. It is a routine integration exercise to derive Equation (1) as an exact gamma-Poisson model specification. ${ }^{(15)}$ The conditions $\alpha \ll \beta$ and $\beta \gg 1$ were then explicitly specified by Teunis and Havelaar (2000) for a valid use of Equation (1) as a dose-response model. ${ }^{(15)}$ Because the random variable $r$ represents probabilities in this context, it should satisfy the condition $0 \leq r \leq 1$ for Equation (1) to be valid as a dose-response model. A gamma distribution defined by Equation (6) has a mean $\mathrm{E}(r)=\frac{\alpha}{\beta}$ and variance $\operatorname{Var}(r)=\frac{\alpha}{\beta^{2}} \cdot{ }^{(21)}$ Therefore, to ensure a high

\footnotetext{
${ }^{1}$ Note that the beta-Poisson model is applied to cases where the dose is assumed to have Poisson error. If the dose is exact, then the proper model (with exponential dose-response parameter beta-distributed across subjects) would be beta-binomial, which has a simple closed-form expression of the dose-response model: $\quad P_{I}\left(d_{i}\right)=$ $1-\frac{\Gamma(\alpha+\beta) \Gamma\left(\beta+d_{i}\right)}{\Gamma(\beta) \Gamma\left(\alpha+\beta+d_{i}\right)}$ where $d_{i}$ is the exact number of organisms that ingested (page 457 in reference 18 . Haas $\mathrm{CN}$. Conditional dose-response relationships for microorganisms: Development and application. Risk analysis. 2002;22(3):455-63.).
} 
probability for $0 \leq r \leq 1$, it requires that $\alpha$ must be much smaller than $\beta$ because if $\alpha>\beta$, it results $\mathrm{E}(r)>1$ and $\operatorname{Var}(\mathrm{r})>>0$. More recently, it was shown in Schmidt et al. (2013) ${ }^{(22)}$ that if $\alpha \ll \beta$ and $\beta \gg 1$ are true, Equation (1) is an accurate approximation for Equation (4) at least in their first and second moment properties (i.e. the mean and variance of the two equations are sufficiently close to each other). However, a critical question remains: how much smaller and larger do these respective conditions have to be for an accurate approximation of Equation (1) for Equation (4) (i.e., of the approximate model for the exact model)? One plausible solution lies in the fact that, by treating Equation (1) as an exact gamma-Poisson model, we are able to estimate the probability of a valid use of the approximate betaPoisson models, given the parameter estimates $\hat{\alpha}$ and $\hat{\beta}$. This is the theoretical ground for the development of the rule-of-thumb for a valid use of Equation (1) and details are given in Section 3.1.

\subsection{Parameter Estimation}

In this paper, the same optimization summary statistic for parameter estimation is used for both the approximate and the exact beta-Poisson models. This is defined as

$$
Y=-2 \sum_{i=1}^{m}\left[y_{i} \log \frac{\pi_{i}}{\pi_{i}^{0}}+\left(N_{i}-y_{i}\right) \log \frac{1-\pi_{i}}{1-\pi_{i}^{0}}\right] .
$$

Note that Equation (7) is a reproduction of Equation 8.33 in Haas et al. (p285(1) $)$ In Equation (7), $m$ is the number of exposure groups in terms of the mean dose level $D_{i} ; N_{i}$ is the total number of individuals in the $i$ th exposure group (total $m$ groups); $y_{i}$ is the number of infected subjects in the $i$ th exposure group; $\pi_{i}$ is the predicted probability of infection of the $i$ th exposure group; and $\pi_{i}^{o}=y_{i} / N_{i}$ is the ratio of the observed number of infected subjects to the number of all subjects at risk at each mean dose level $D_{i}$ (see Table I for the notation and definition details). The summary statistic $Y$ defined by Equation (7) is a measure of the discrepancy between the observed responses $\left(\pi_{i}^{o}\right)$ and the model predicted responses $\left(\pi_{i}\right)$ of the dose-response data. ${ }^{(23)}$ Note that if estimating $\pi_{i}=P_{I}(d)$ from Equation (1) and then substituting the results into Equation (7), the maximum likelihood estimates (MLEs) of the model parameters, $\hat{\alpha}$ and $\hat{\beta}$, are obtained for the approximate beta-Poisson model by minimizing $Y^{(1,24)}$; if, 
instead, estimating $\pi_{i}=P_{I}(d)$ from Equation (4) and then substituting the results into Equation (7), $\hat{\alpha}_{e}$ and $\hat{\beta}_{e}$, the MLEs of the model parameters of the exact beta-Poisson model will be obtained.

The evaluation of the Kummer confluent hypergeometric function (Equation (5)) is non-trivial but can be obtained by using the $\mathrm{R}^{(25)}$ function 'hyperg_1F1' in the R package 'gsl'. (26) The built-in optimization function 'optim' in R is employed to obtain the MLEs of $\alpha$ and $\beta$ that minimize $Y$ for both the approximate and the exact beta-Poisson models ${ }^{2}$.

\subsection{Datasets}

The QMRA Wiki ${ }^{(2)}$ presents 138 feeding trial experimental dose-response datasets and each of them was fitted either by an exponential dose-response model ( Equation (2)) or by an approximate beta-Poisson dose-response model (Equation (1)). Of these 138 datasets, 53 are best fitted by the exponential model and 85 are best fitted by the approximate beta-Poisson model as presented in the QMRA Wiki ${ }^{(2)}$. The exact beta-Poisson model (Equation (4)) was not used for fitting the dose-response data in the QMRA $\mathrm{Wiki}^{(2)}$. It is these 85 datasets used for fitting the approximate beta-Poisson dose-response model in the QMRA Wiki ${ }^{(2)}$ are examined in this study for validating the proposed rule-of-thumb. Due to the space limitation, only the descriptions for each of these 85 datasets are provided in this paper (Table III in the appendix). Readers are reminded that the data analysis results produced from this study are based on the data details presented in the QMRA Wiki ${ }^{(2)}$. For the originality and the correctness of these datasets readers should refer to the original references provided in the QMRA Wiki(2).

\section{A RULE-OF-THUMB FOR THE APPROXIMATE BETA-POISSON MODEL}

\subsection{Development Of The Rule-of-thumb}

The theoretical ground for the development of the rule-of-thumb for a valid use of the approximate betaPoisson model (Equation (1)) lies in the fact that Equation (1) can be derived as the exact gamma-Poisson

\footnotetext{
${ }^{2}$ The R source code for repeating the analysis results presented in this paper is available on request by contacting the corresponding author.
} 
model in which the random variable $r$, being assumed as a probability in the dose-response analysis (as defined in Table I), follows a gamma distribution which cannot guarantee $0<r \leq 1$. Therefore, to ensure a valid application of Equation (1) as a dose-response model, the model parameters $\alpha$ and $\beta$ should be confined to an admissible range/area such that $\operatorname{Pr}(0<\mathrm{r}<1)$ is very close to one; for example, this may be arbitrarily defined as $\operatorname{Pr}(0<\mathrm{r}<1)>0.99$. This probability measure, $\operatorname{Pr}(0<\mathrm{r}<1)$, is defined as:

$$
\operatorname{Pr}(0<r<1 \mid \alpha, \beta)=\int_{0}^{1} f(r \mid \alpha, \beta) d r,
$$

where $f(r \mid \alpha, \beta)$ is the gamma distribution pdf defined by Equation (6). Equation (8) can be evaluated easily using a standard statistical software program (e.g., the built-in $\mathrm{R}^{(25)}$ function 'pgamma'). For example, $\operatorname{Pr}(0<r<1 \mid \alpha=1, \beta=4.63$ ) is 0.9902 (up to 4 decimal places (dp) accuracy).

In QMRA practice, the model parameters $\alpha$ and $\beta$ are unknown and the MLEs are accepted as the valid estimates of $\alpha$ and $\beta$ for the estimation of the probability $\operatorname{Pr}(0<\mathrm{r}<1 \mid \alpha, \beta)$ in Equation (8). Since the purpose of developing this rule-of-thumb is for facilitating the evaluation of Equation (4), it is the MLEs obtained from Equation (1), $\hat{\alpha}$ and $\hat{\beta}$, will be used so that Equation (8) can be evaluated as $\operatorname{Pr}(0<r$ $<1 \mid \hat{\alpha}, \hat{\beta})=\int_{0}^{1} f(r \mid \hat{\alpha}, \hat{\beta}) d r$. Based on the arbitrarily defined criterion for a good approximation, $\operatorname{Pr}(0<\mathrm{r}$ $<1)>0.99$, therefore, the goal for developing a rule-of-thumb is to elicit a specification of the admissible range/area which ideally should cover all the possible pairs of $\hat{\alpha}$ and $\hat{\beta}$ so that any identified valid application of Equation (1) should satisfy the condition $\operatorname{Pr}(0<r<1 \mid \hat{\alpha}, \hat{\beta})>0.99$ and any application which satisfies the condition $\operatorname{Pr}(0<r<1 \mid \hat{\alpha}, \hat{\beta})>0.99$ should be identified as a valid application (i.e., the rule-of-thumb can be applied as a sufficient and necessary condition).

The relationships between the probability $\operatorname{Pr}(0<r<1)$ and model parameters $\alpha$ and $\beta$ are examined in Fig. 1 for determination of the constraint conditions in the rule-of-thumb. In Fig. 1(a), represented by four black solid curves, four levels of the probability contour lines (evaluated at $\operatorname{Pr}(0<r<1)=0.8$, 0.90, 0.99, and 0.999, respectively) are drawn against the corresponding paired $\alpha$ and $\beta$ values. For meeting the $\operatorname{Pr}(0<\mathrm{r}<1)>0.99$ criterion, the admissible paired $\alpha$ and $\beta$ values should be confined to the area above the $\operatorname{Pr}(0<r<1)=0.99$ contour line. As shown in Fig. $1(\mathrm{a})$, the $\operatorname{Pr}(0<r<1)=0.99$ 
contour line starts from the origin and increases monotonically and smoothly along the horizontal axis (i.e., $\alpha$ is positively correlated with $\beta$ in terms of the probability contour lines). The increase appears very linear for $2 \leq \alpha<20$ in Fig. 1(a). It is therefore likely that the contour line can be best approximated using a piece-wisely fitted curve, e.g., by a power function (or power laws ${ }^{(27)}$ ) of the form $\beta=(\mathrm{A} \alpha)^{\mathrm{B}}$ for $\alpha<2$ and by a linear function (i.e., a straight line) of the form $\beta=\mathrm{A} \alpha+\mathrm{B}$ for $2 \leq \alpha<$ 20, where A and B are parameters to be determined by best matching the contour line. As found in the QMRA literature, all reported $\hat{\alpha}$ values are less than two (ranged from 0.03 to 1.76$).{ }^{(2,13,28)}$ Therefore, it is reasonable for the rule-of-thumb to primarily focus on $\alpha<2$ so that all practically feasible $\hat{\alpha}$ values are most likely included. Plot (b) is an enlargement of the bottom-left corner of plot (a) where $\alpha<2$ and $\beta<10$. The bold red dashed lines are defined by the power function $\beta=(22 \alpha)^{0.50}$ for $\alpha<2$ and the linear function $\beta=1.4 \alpha+4.4$ for $2 \leq \alpha<20$. The piece-wise curve fitting details can be found in the appendix. The shaded area represents all the paired $\alpha$ and $\beta$ values which satisfy the condition $\beta>$ $(22 \alpha)^{0.50}$. Fig. 1(b) shows clearly that the red dashed lines are a very good approximation to the $\operatorname{Pr}(0<r<1)=0.99$ contour line. 
(a)

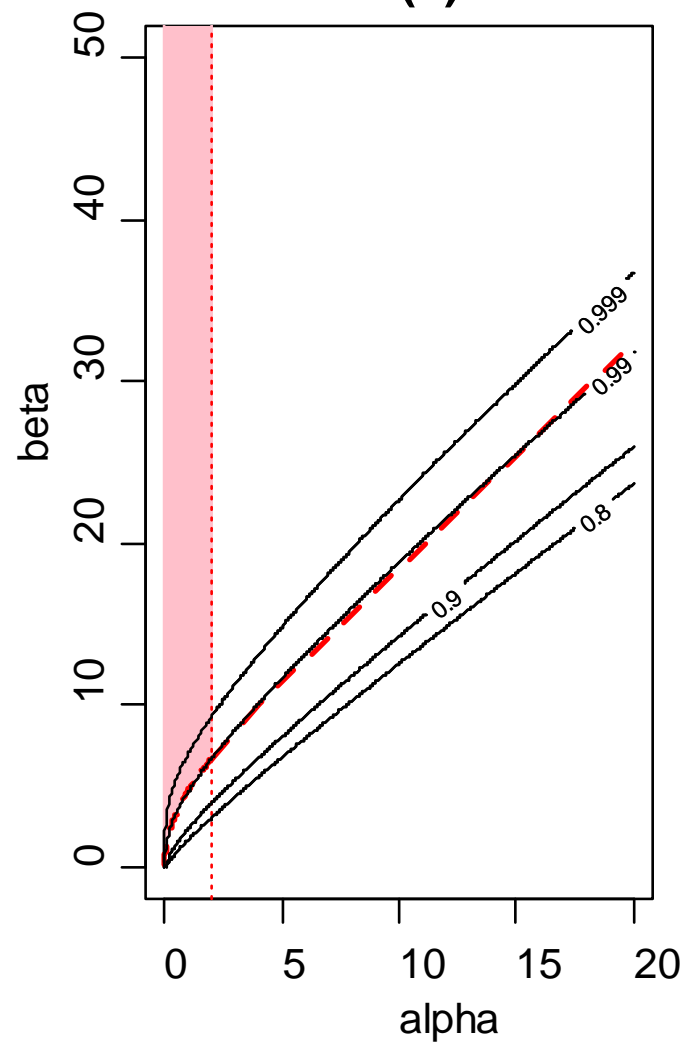

(b)

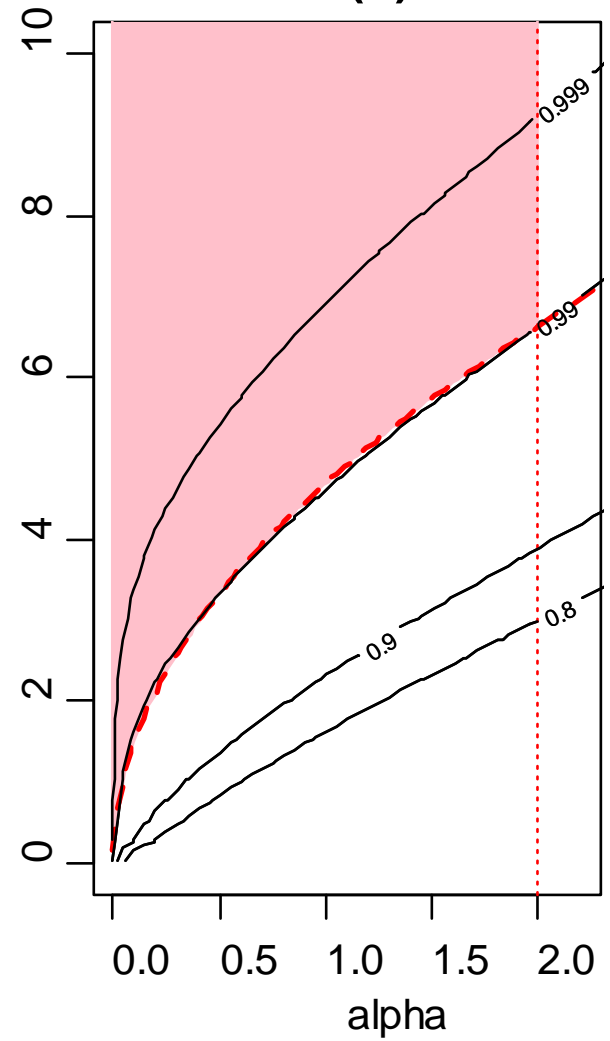

Fig. 1. Relationships between the probability $\operatorname{Pr}(0<r<1)$ and model parameters $\alpha$ and $\beta$ : The black solid curves are the contour lines of different levels of the probability $\operatorname{Pr}(0<r<1)$ evaluated with respect to the corresponding paired $\alpha$ and $\beta$ values ( $\alpha$ is on the horizontal axis and $\beta$ is on the vertical axis). The bold red dashed lines are defined by the power function $\beta=(22 \alpha)^{0.50}$ for $\alpha<2$ and the linear function $\beta=1.4 \alpha+4.4$ for $2 \leq \alpha<20$. The red vertical dotted lines are drawn at the position $\alpha=2$. Plot (b) is an enlargement of the bottom-left corner of plot (a) where $\alpha<2$ and $\beta<10$. The shaded areas represent all the paired $\alpha$ and $\beta$ values which satisfy the condition $\beta>(22 \alpha)^{0.50}$.

Because the probability contour lines are not defined in a closed mathematical form, the best-fit piecewise curve does not perfectly match the 0.99 probability contour line; even so, however, the approximation is indeed very good as shown in Fig. 1. In particular, it can easily be shown, numerically, that $0.988<\operatorname{Pr}(0<r<1 \mid \alpha, \beta)<0.992$ for $0.02<\alpha<2$ and $\beta=(22 \alpha)^{0.50}$. Therefore, practically, 
the condition $\beta>(22 \alpha)^{0.50}$ could be considered as a sufficient and necessary condition to ensure $\operatorname{Pr}(0<r<1 \mid \alpha, \beta)>0.99$ (2 dp) for $0.02<\alpha<2$. By taking into account both the theoretical justification and the QMRA data analysis empirical evidence, a rule-of-thumb for a valid use of Equation (1) is therefore specified as:

i. $\quad$ obtain the parameter estimates $\hat{\alpha}$ and $\widehat{\beta}$ from Equation (1);

ii. if and only if the resulting parameter estimates satisfy the condition $\hat{\beta}>(22 \hat{\alpha})^{0.50}$ for $0.02<$ $\hat{\alpha}<2$, Equation (1) is considered a valid application as an accurate approximation to Equation (4).

In comparison with the general rule $\alpha \ll \beta$ and $\beta \gg 1$, this rule-of-thumb has actually relaxed the $\beta \gg 1$ condition and specified a specific condition about the $\alpha$ and $\beta$ values for one to apply. The proposed rule-of-thumb therefore provides a practical and better guidance for the valid use of the approximate betaPoisson model in dose-response analysis. To the best of our knowledge, the $0.02<\hat{\alpha}<2$ range has covered all the practically feasible $\hat{\alpha}$ values for dose-response analysis reported in the literature so far. The very small curve matching errors, i.e., $\operatorname{Pr}(0<r<1 \mid \alpha, \beta)=0.99 \pm 0.002$ based on the rule-ofthumb condition, should not have any practically significant impact on the use of the rule-of-thumb as a sufficient and necessary condition for assessing the validity or the approximation performance of the approximate beta-Poisson model. This statement is supported by the fact that, for the total 85 models examined in this study, 17 models are identified as invalid approximation application models and the other 68 models are valid models; without exception, all invalid models clearly have the probabilities $\operatorname{Pr}(0<r<1 \mid \hat{\alpha}, \hat{\beta})<0.99$ and all valid models are clearly with $\operatorname{Pr}(0<r<1 \mid \hat{\alpha}, \hat{\beta})>0.99$ as detailed in Table II in Section 4.1.

For $\hat{\alpha} \leq 0.02$ or $\hat{\alpha} \geq 2$ cases, if future QMRA practice does show a demand for the guidelines to better cover these cases, the proposed rule-of-thumb can always be revised following the methodology described in this section with or without any new dose-response data analysis evidences. For example, it 
has been shown in Fig. 1(a) that for the $2 \leq \alpha<20$ cases, the 0.99 probability contour line can be closely approximated by the linear function $\beta=1.4 \alpha+4.4$.

It is worth noting that, by following the same development approach described above, one could specify a different rule-of-thumb based on a criterion of one's choice, e.g., $\operatorname{Pr}(0<r<1 \mid \hat{\alpha}, \hat{\beta})>0.95$ or $\operatorname{Pr}(0<r$ $<1 \mid \hat{\alpha}, \hat{\beta})>0.999$ for the purpose of approximation performance assessment.

This rule-of-thumb is supposed to ensure an accurate approximation in terms of matching the doseresponse curves produced by Equation (1) and Equation (4). However, if the approximation performance is assessed by taking into account the uncertainty in the model parameter estimation, other factors are involved, such as the number of the data points and how the mean dose range has been covered by these data points. Therefore, the performance of the rule-of-thumb by matching the confidence bands about the dose-response curves should also be assessed. For a better understanding and interpretation of the validation results, the procedure/methodology of how to validate this rule-of-thumb using empirical dose response datasets is described next in subsection 3.2 before the presentation and discussions of the validation results in Section 4.

\subsection{Validation Methodology For The Rule-of-thumb}

The rule-of-thumb should be validated by application to as many as possible of the completed betaPoisson models related to experimental dose-response data in QMRA practice (e.g., those datasets listed in Table III). The first step in the validation process is to fit both the approximate and the exact betaPoisson models to each of the 85 datasets by following the parameter estimation procedure described in Section 2.3. Therefore, there are 85 sets of model parameter estimates obtained in this study (i.e., 85 sets of $\hat{\alpha}, \widehat{\beta}, \hat{\alpha}_{e}, \hat{\beta}_{e}$ values). The second step is to calculate the probability $\operatorname{Pr}(0<r<1 \mid \hat{\alpha}, \hat{\beta})$ for a valid application of Equation (1) for each of the 85 approximate models. The details of how the probability $\operatorname{Pr}(0<r<1 \mid \hat{\alpha}, \hat{\beta})$ is evaluated can be found in the previous section. Step 3, apply the rule-of-thumb, $\hat{\beta}>$ 
(22 $\hat{\alpha})^{0.50}$, to identify the valid approximate models from those invalid ones. All analysis results obtained from Step 1 to Step 3 can be summarised in one table for easy comparison and interpretation. Based on the analysis results from Step 1 to Step 3, the probability of infection, $P_{I}(d)$, can be estimated from Equation (1) (the approximate model) or from Equation (4) (the exact model). The approximation performance of Equation (1) is then assessed by drawing and superposing the dose-response curves of Equations (1) and (4) in one plot for each dataset as the Step 4 of the validation process. In the plots for matching the dose-response curves, the vertical axis measures the probability of infection ranged from zero to unity; the horizontal axis measures the mean dose (on the natural log scale) ranged from $10^{-3}$ (0.001) to $10^{11}(100,000,000,000)$ and 42 mean dose levels roughly evenly-spaced within the range are arbitrarily determined as the horizontal-axis plotting position for the dose-response curve. Because the horizontal-axis measures the mean dose on the natural log scale, any visually identifiable discrepancies between the approximate model curve and the exact model curve is considered as a good/decision-making evidence for an invalid application of Equation (1). Finally (Step 5), among the valid application models, for those datasets which have been identified as sensitive to the sampling variation, the confidence bands of the dose-response curves are plotted and superposed by the data points (the fractions of the infected subjects) to examine the impact of the uncertainty in parameter estimation. As it is well accepted in QMRA practice, a parametric bootstrap algorithm ${ }^{(29)}$ is employed to construct the $95 \%$ confidence bands for the dose-response curves. ${ }^{(1,2,13,15)}$ The validation analysis on the rule-of-thumb is conducted by following the process as described above and the validation results and the discussions are presented in the next section.

\section{VALIDATION RESULTS AND DISCUSSION}

The validation process described in Section 3.2 is followed for validating the rule-of-thumb with respect to all 85 datasets listed in Table III. The performance of the rule-of-thumb is first examined by matching the corresponding dose-response curves produced from Equation (1) and Equation (4) and these validation results are presented in Section 4.1. Since a good match in dose-response curves does not 
guarantee a good match for the confidence bands if the $\hat{\beta}$ value in the model is not large enough (e.g., $\hat{\beta}$ $<100){ }^{(15,22)}$ those valid approximate beta-Poisson models with datasets which are sensitive to the sampling variations are then identified and examined for the impact of the uncertainty in parameter estimation as presented in Section 4.2.

\subsection{Validation Of The Rule-of-thumb By Matching The Dose-Response Curves}

The numeric outputs of the validation results are summarised in Table II which contains the dataset serial numbers in column 1 (corresponding to the datasets described in table III); the approximate model MLEs $\hat{\alpha}$ and $\hat{\beta}$ in columns 2 and 3; the exact model MLEs $\hat{\alpha}_{e}$ and $\hat{\beta}_{e}$ in columns 4 and 5; the rule of thumb assessment result (i.e., satisfied or not) in column 6; and the validity measure $\operatorname{Pr}(0<r<1 \mid \hat{\alpha}, \hat{\beta})$ results in column 7. As shown in Table II, the first 17 approximate beta-Poisson models (those models indicated by 'No' in column 6) are identified as invalid to apply for QMRA dose-response analysis.

Correspondingly, it shows that the $\operatorname{Pr}(0<r<1 \mid \hat{\alpha}, \hat{\beta})$ values for models 1 to 17 are much lower than those $\operatorname{Pr}(0<r<1 \mid \hat{\alpha}, \hat{\beta})$ values obtained from models 18 to 85 which are identified as valid application cases. All 17 invalid models have a $\operatorname{Pr}(0<r<1 \mid \hat{\alpha}, \hat{\beta})$ value less than 0.99 . Of the 68 valid models (related to datasets 18 to 85), 65 models have the probability $\operatorname{Pr}(0<r<1 \mid \hat{\alpha}, \hat{\beta})>0.9999$, and the model related to dataset 42 has a probability $\operatorname{Pr}(0<r<1 \mid \hat{\alpha}, \hat{\beta})=0.9990(4 \mathrm{dp})$; the model related to dataset 72 has a probability $\operatorname{Pr}(0<r<1 \mid \hat{\alpha}, \hat{\beta})=0.9996$ (4 dp), and the model related to dataset 78 has a probability $\operatorname{Pr}(0<$ $r<1 \mid \hat{\alpha}, \hat{\beta})=0.9971(4 \mathrm{dp})$.

Based on the numeric validation results presented in Table II, one is able to draw dose-response curves to examine any visually identifiable discrepancies between the approximate model and the exact model. The dose-response curves matching results for models 1 to 17 (i.e., all invalid approximate models) are displayed in Fig. 2 and the dose-response curves matching results for models 18 to 85 (i.e., all valid approximate models) are displayed in Fig. 3. 
Table II. The numeric validation results based on the applications of the rule-of-thumb to the 85 experimental dose-response datasets: $\hat{\alpha}$ and $\hat{\beta}$ are the MLEs obtained from the approximate beta-Poisson model; $\hat{\alpha}_{e}$ and $\hat{\beta}_{e}$ are the corresponding MLEs obtained from the exact beta-Poisson model.

\begin{tabular}{|c|c|c|c|c|c|c|}
\hline Dataset & $\hat{\alpha}$ & $\hat{\beta}$ & $\hat{\alpha}_{e}$ & $\hat{\beta}_{e}$ & $\begin{array}{l}\text { Does } \hat{\beta} \text { meet the condition } \\
\hat{\beta}>(22 \hat{\alpha})^{0.50} \text { ? }\end{array}$ & $\begin{array}{l}\operatorname{Pr}(0<r<1 \mid \hat{\alpha}, \hat{\beta}) \\
(4 \mathrm{dp})\end{array}$ \\
\hline 1 & 0.0579 & 0.00305 & 0.0579 & 0.155 & No & 0.7373 \\
\hline 2 & 0.145 & 0.421 & 0.145 & 0.766 & No & 0.8986 \\
\hline 3 & 0.210 & 1.92 & 0.210 & 2.19 & No & 0.9847 \\
\hline 4 & 0.225 & 0.157 & 0.149 & 0.152 & No & 0.7030 \\
\hline 5 & 0.201 & 0.303 & 0.158 & 0.301 & No & 0.8169 \\
\hline 6 & 0.252 & 0.261 & 0.186 & 0.222 & No & 0.7483 \\
\hline 7 & 0.701 & 0.112 & 0.0338 & 0.00100 & No & 0.2266 \\
\hline 8 & 0.181 & 0.498 & 0.152 & 0.484 & No & 0.8905 \\
\hline 9 & 0.200 & 0.337 & 0.176 & 0.429 & No & 0.8312 \\
\hline 10 & 0.182 & 0.312 & 0.153 & 0.379 & No & 0.8375 \\
\hline 11 & 0.253 & 0.426 & 0.167 & 0.192 & No & 0.8214 \\
\hline 12 & 0.0728 & 0.108 & 0.0364 & 0.0866 & No & 0.8769 \\
\hline 13 & 0.096 & 0.0701 & 0.0198 & 0.0200 & No & 0.8081 \\
\hline 14 & 0.270 & 1.39 & 0.264 & 1.60 & No & 0.9572 \\
\hline 15 & 0.114 & 1.05 & 0.114 & 1.44 & No & 0.9743 \\
\hline 16 & 0.145 & 1.51 & 0.145 & 1.91 & No & 0.9829 \\
\hline 17 & 0.101 & 0.353 & 0.0877 & 0.469 & No & 0.9186 \\
\hline 18 & 0.549 & 11,200 & 0.549 & 11,200 & Yes & $>0.9999$ \\
\hline 19 & 0.328 & 746 & 0.328 & 747 & Yes & $>0.9999$ \\
\hline 20 & 0.213 & 19.2 & 0.213 & 19.4 & Yes & $>0.9999$ \\
\hline 21 & 0.267 & 20.5 & 0.267 & 20.7 & Yes & $>0.9999$ \\
\hline 22 & 0.265 & 179 & 0.265 & 180 & Yes & $>0.9999$ \\
\hline 23 & 0.144 & 7.36 & 0.144 & 7.77 & Yes & $>0.9999$ \\
\hline 24 & 0.319 & 8,600 & 0.319 & 8,596 & Yes & $>0.9999$ \\
\hline 25 & 0.117 & 85.0 & 0.117 & 84.8 & Yes & $>0.9999$ \\
\hline 26 & 0.166 & 1,935 & 0.166 & 1,937 & Yes & $>0.9999$ \\
\hline 27 & 0.357 & $82,450,000$ & 0.357 & $82,510,000$ & Yes & $>0.9999$ \\
\hline 28 & 0.487 & 189,300 & 0.487 & 189,400 & Yes & $>0.9999$ \\
\hline 29 & 0.155 & 24,400 & 0.155 & 24,400 & Yes & $>0.9999$ \\
\hline 30 & 0.086 & 57.4 & 0.086 & 57.0 & Yes & $>0.9999$ \\
\hline 31 & 0.263 & 273,000 & 0.263 & 273,000 & Yes & $>0.9999$ \\
\hline 32 & 0.250 & $6,040,000$ & 0.250 & $6,040,000$ & Yes & $>0.9999$ \\
\hline 33 & 0.178 & $1,780,000$ & 0.178 & $1,780,000$ & Yes & $>0.9999$ \\
\hline 34 & 0.221 & $3,120,000$ & 0.221 & $3,110,000$ & Yes & $>0.9999$ \\
\hline 35 & 0.0755 & 175.2 & 0.0755 & 175.4 & Yes & $>0.9999$ \\
\hline
\end{tabular}




\begin{tabular}{|c|c|c|c|c|c|c|}
\hline 36 & 0.206 & $4,630,000$ & 0.206 & $4,630,000$ & Yes & $>0.9999$ \\
\hline 37 & 0.162 & $1,410,000$ & 0.162 & $1,410,000$ & Yes & $>0.9999$ \\
\hline 38 & 0.375 & 33,300 & 0.375 & 33,400 & Yes & $>0.9999$ \\
\hline 39 & 0.269 & $6,570,000$ & 0.269 & $6,580,000$ & Yes & $>0.9999$ \\
\hline 40 & 0.253 & 19.1 & 0.253 & 19.3 & Yes & $>0.9999$ \\
\hline 41 & 0.695 & 1,984 & 0.695 & 1,983 & Yes & $>0.9999$ \\
\hline 42 & 0.120 & 3.52 & 0.117 & 3.51 & Yes & 0.9990 \\
\hline 43 & 0.172 & 37,600 & 0.172 & 37,500 & Yes & $>0.9999$ \\
\hline 44 & 0.0422 & 132 & 0.0422 & 133 & Yes & $>0.9999$ \\
\hline 45 & 0.0400 & 24.3 & 0.0400 & 24.8 & Yes & $>0.9999$ \\
\hline 46 & 0.0936 & 28,300 & 0.0936 & 28,300 & Yes & $>0.9999$ \\
\hline 47 & 0.190 & 494 & 0.190 & 494 & Yes & $>0.9999$ \\
\hline 48 & 0.355 & 1,086 & 0.355 & 1,086 & Yes & $>0.9999$ \\
\hline 49 & 0.109 & 256 & 0.109 & 256 & Yes & $>0.9999$ \\
\hline 50 & 0.673 & 10,700 & 0.673 & 10,700 & Yes & $>0.9999$ \\
\hline 51 & 0.549 & 4,460 & 0.549 & 4,460 & Yes & $>0.9999$ \\
\hline 52 & 0.601 & 6,820 & 0.600 & 6,810 & Yes & $>0.9999$ \\
\hline 53 & 0.777 & 14.8 & 0.770 & 14.3 & Yes & $>0.9999$ \\
\hline 54 & 0.866 & 15.6 & 0.860 & 15.1 & Yes & $>0.9999$ \\
\hline 55 & 0.675 & 13.2 & 0.667 & 12.7 & Yes & $>0.9999$ \\
\hline 56 & 0.175 & 21,700 & 0.175 & 21,700 & Yes & $>0.9999$ \\
\hline 57 & 0.111 & 6,610 & 0.111 & 6,590 & Yes & $>0.9999$ \\
\hline 58 & 0.203 & 29,200 & 0.203 & 29,200 & Yes & $>0.9999$ \\
\hline 59 & 0.318 & 4,720 & 0.318 & 4,730 & Yes & $>0.9999$ \\
\hline 60 & 0.291 & 4,510 & 0.291 & 4,510 & Yes & $>0.9999$ \\
\hline 61 & 0.230 & 253 & 0.230 & 253 & Yes & $>0.9999$ \\
\hline 62 & 0.389 & 3,400 & 0.389 & 3,400 & Yes & $>0.9999$ \\
\hline 63 & 0.885 & 441,000 & 0.885 & 441,000 & Yes & $>0.9999$ \\
\hline 64 & 0.0621 & 492 & 0.0621 & 492 & Yes & $>0.9999$ \\
\hline 65 & 0.108 & 15,500 & 0.108 & 15,500 & Yes & $>0.9999$ \\
\hline 66 & 0.265 & 117 & 0.265 & 117 & Yes & $>0.9999$ \\
\hline 67 & 0.277 & 21.1 & 0.275 & 21.0 & Yes & $>0.9999$ \\
\hline 68 & 0.143 & 284 & 0.143 & 285 & Yes & $>0.9999$ \\
\hline 69 & 0.135 & 18.5 & 0.135 & 18.7 & Yes & $>0.9999$ \\
\hline 70 & 0.157 & 9.17 & 0.156 & 9.26 & Yes & $>0.9999$ \\
\hline 71 & 0.117 & 9.87 & 0.117 & 10.3 & Yes & $>0.9999$ \\
\hline 72 & 0.110 & 4.30 & 0.11 & 4.66 & Yes & 0.9996 \\
\hline 73 & 0.250 & 16.2 & 0.250 & 16.1 & Yes & $>0.9999$ \\
\hline 74 & 0.198 & $19,860,000$ & 0.198 & $19,860,000$ & Yes & $>0.9999$ \\
\hline 75 & 0.318 & 870 & 0.318 & 869 & Yes & $>0.9999$ \\
\hline 76 & 0.110 & 70,400 & 0.110 & 70,400 & Yes & $>0.9999$ \\
\hline 77 & 0.131 & $14,800,000$ & 0.131 & $14,900,000$ & Yes & $>0.9999$ \\
\hline 78 & 0.0794 & 2.31 & 0.0784 & 2.58 & Yes & 0.9971 \\
\hline
\end{tabular}




\begin{tabular}{lllllll}
79 & 1.06 & 995 & 1.06 & 992 & Yes & $>0.9999$ \\
80 & 0.374 & 187 & 0.373 & 187 & Yes & $>0.9999$ \\
81 & 0.581 & 412,000 & 0.581 & 412,000 & Yes & $>0.9999$ \\
82 & 0.903 & $1,080,000$ & 0.903 & $1,080,000$ & Yes & $>0.9999$ \\
83 & 0.429 & 165,000 & 0.429 & 165,000 & Yes & $>0.9999$ \\
84 & 0.137 & 93.2 & 0.137 & 93.2 & Yes & $>0.9999$ \\
85 & 1.76 & 214,000 & 1.76 & 214,000 & Yes & $>0.9999$ \\
\hline
\end{tabular}

In Figures 2 and 3, the exact beta-Poisson curves are represented by black solid lines and the approximate beta-Poisson curves are drawn in red solid lines. For each plot, the approximate model's dose-response curve is first drawn and then the exact model's curve is superimposed upon the approximate curve. Therefore, if there are two colours or two curves visually identifiable in a plot, it is considered as a definite evidence of an unacceptable approximation case. For models related to datasets 1 to 17 (the 17 invalid models) displayed in Fig. 2, the majority of the plots (e.g., 13 models related to datasets 1, 2, 4 through to 13, and 17) have showed substantial discrepancy patterns. By looking at the numeric results in column 7 of Table II, it shows that $\operatorname{Pr}(0<r<1 \mid \hat{\alpha}, \hat{\beta})<0.92$ for these 13 models. In contrast, for models related to datasets 18 through to 85 displayed in Fig.3 (the 68 valid models), the exact curves and approximate curves have matched almost perfectly. Only very few tiny red bits and pieces appear in the dose-response curves in a handful of plots (e.g., models related to datasets 21, 25, 30, 42, 69, 71, and 78) if these plots are displayed in colour (or otherwise no discrepancies are visually identifiable). It is noted that four invalid models in Fig. 2 (related to datasets 3, 14, 15, and 16) have also showed a reasonably good match in the dose-response curves because their probability $\operatorname{Pr}(0<r<1 \mid \hat{\alpha}, \hat{\beta})$ values are all greater than 0.95.

One thing seems having been ignored in the literature is the impact on the calculation of $\mathrm{N}_{50}$, the median effective (mean) dose level, in application of the approximate beta-Poisson model. By definition, $\mathrm{N}_{50}$ is derived from the equation $P_{I}\left(d=\mathrm{N}_{50}\right)=0.5$ using those parameter estimates obtained from Equation (1) (in the approximate beta-Poisson model case) or Equation (4) (in the exact beta-Poisson model case), accordingly. It is important to note that the formula 


$$
\mathrm{N}_{50}=\beta\left(2^{\frac{1}{\alpha}}-1\right)
$$

is derived from Equation (1). Therefore, this formula (Equation (9)) is only applicable for calculating $\mathrm{N}_{50}$ in the approximate beta-Poisson model cases. It could be very misleading if one applies Equation (9) using the exact model parameter estimates. For example, for dataset 11 (rotavirus experiment 70 data, Table IV), the model parameter estimates are $\hat{\alpha}=0.253$ and $\hat{\beta}=0.426$, and $\hat{\alpha}_{e}=0.167$ and $\hat{\beta}_{e}=$ 0.192 (Table II) and a visually identifiable deviation is found in the dose-response curves (Fig. 2). By applying Equation (9) using the exact beta-Poisson model parameter estimates, one gets $\mathrm{N}_{50}=\hat{\beta}_{e}\left(2^{\frac{1}{\hat{\alpha}_{e}}}-\right.$ 1) $=$ 12.0. In fact, the correct answer is $N_{50}=2.55$ for the exact model - an iterative numeric calculation algorithm needs to be employed in evaluating Equation (4) for obtaining this result. In comparison, $\mathrm{N}_{50}=\hat{\beta}\left(2^{\frac{1}{\hat{\alpha}}}-1\right)=6.17$ is the result for the approximate model related to this dataset. Essentially, the $\mathrm{N}_{50}$ calculation pitfall is associated with the validity of Equation (1). This dataset 11 example shows that an invalid application of the approximate beta-Poisson model could result in a substantial underestimation of the estimated level of risk (i.e., an over-estimation of $\mathrm{N}_{50}$ ). The validation results presented above provide strong evidence to justify the effectiveness of the probability $\operatorname{Pr}(0<r<1 \mid \hat{\alpha}, \hat{\beta})$ as a validity measure and the rule-of-thumb $\hat{\beta}>(22 \hat{\alpha})^{0.50}$ for ensuring an accurate approximation to the exact dose-response curve in QMRA practice. As noted in Teunis and Havelaar (2000) ${ }^{(15)}$ and Schmidt et al. (2013) ${ }^{(22)}$, a near perfect match in doseresponse curves does not guarantee a good match in confidence bands (i.e., when uncertainty in model parameters is taken into account). Importantly, those valid models with $\hat{\beta}$ values less than 100 are likely to have a poor confidence band. Not surprisingly, those a handful valid models which have showed some subtle discrepancy signs in dose-response curves, i.e., models related to datasets 21, 25, 30, 42, 69, 71, and 78, all fall into this category, i.e., their $\hat{\beta}$ values are all less than 100 (column 3, Table II). The impacts of the uncertainty in parameter estimation for these models are examined next in Section 4.2. 


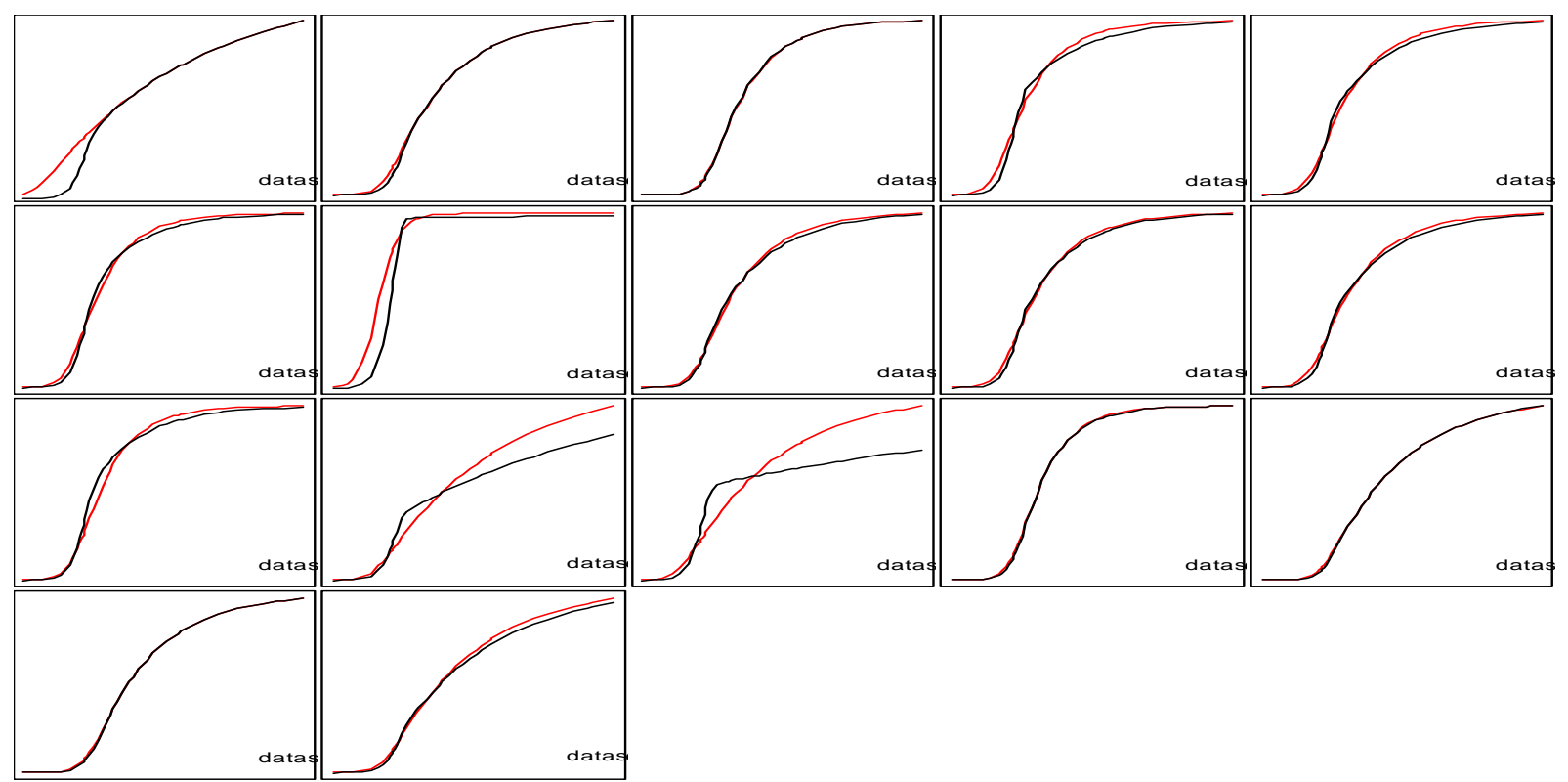

Fig. 2. Validation of the rule-of-thumb by matching the dose-response curves produced by the approximate and exact beta-Poisson models based on parameter estimates presented in Table II: datasets 1 to 17. The exact beta-Poisson curves are represented by black solid lines; the approximate beta-Poisson curves are red solid lines. The vertical axis measures the probability of infection ranged from zero to unity; the horizontal axis measures the mean dose (on the natural log scale) ranged from $10^{-3}(0.001)$ to $10^{11}$ $(100,000,000,000)$. 


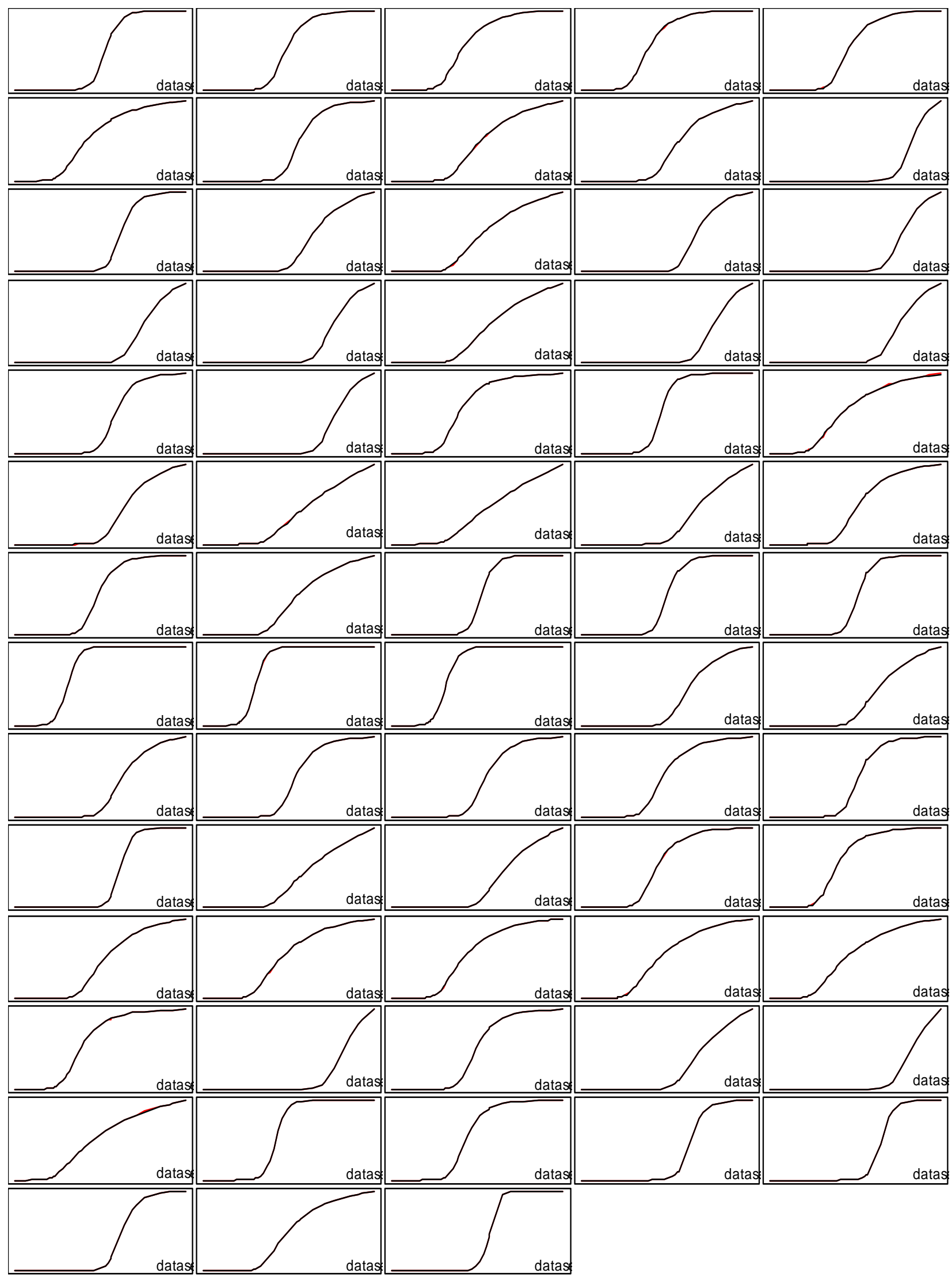


Fig. 3. Validation of the rule-of-thumb by matching the dose-response curves produced by the approximate and exact beta-Poisson models based on parameter estimates presented in Table II: datasets 18 to 85 . The exact beta-Poisson curves are represented by the black solid lines; the approximate betaPoisson curves by the red solid lines. The vertical axis measures the probability of infection ranged from zero to unity; the horizontal axis measures the mean dose (on the natural log scale) ranged from $10^{-3}$ (0.001) to $10^{11}(100,000,000,000)$.

\subsection{Validation Of The Rule-of-thumb By Taking Into Account Uncertainty}

Among the 68 valid approximate beta-Poisson models (related to datasets 18 through to 85), nine models (related to datasets $21,23,30,42,54,55,70,73$, and 78) are identified as having poor confidence bands, i.e., the approximate confidence band deviates from the exact model's confidence band badly or goes beyond the maximum risk dose-response curve (defined and calculated as $P_{I}(d)=1-\exp (-d)$, i.e., Equation (2) with $r=1$ ). ${ }^{(15)}$ Different factors may have contributed to the bad-match confidence band performance for a valid approximate model. For identifying the possible influence factors, one model (related to dataset 67) with $\hat{\beta}<<100$ but with relatively good-match confidence band performance is included in the validation analysis in this section for comparison. A parametric bootstrap algorithm ${ }^{(29)}$ is employed to construct the 95\% confidence bands for the dose-response curves for both the approximate and the exact models. Therefore, 10 models (related to datasets 21, 23, 30, 42, 54, 55, 67, 70, 73, and 78) are selected for validation of the rule-of-thumb by taking into account the uncertainty in parameter estimation and the outcomes are graphically shown in Fig. 4. These 10 datasets are cited from the QMRA $\mathrm{Wiki}^{(2)}$ and the details are provided in Table IV in the appendix.

In Fig. 4, the bold red dashed lines represent the maximum risk dose-response curve. This is a limiting case for beta-Poisson dose-response models where the curve obtained from the exact model (Equation 4) can never go beyond this maximum risk dose-response curve, even when parameter uncertainty is taken into account. ${ }^{(15)}$ This allows the performance of the approximate model (Equation 1 ) to be examined by 
identifying any cases where the 95\% confidence bands of the exact and the approximate models do not match or where the upper bound curves of the approximate models go beyond the maximum risk doseresponse curves. In cases where these maximum risk dose-response curves are exceeded, it is implied that the approximate model is predicting more illnesses than actual exposures (i.e., the probability of infection exceeds the probability of ingesting any organisms). 

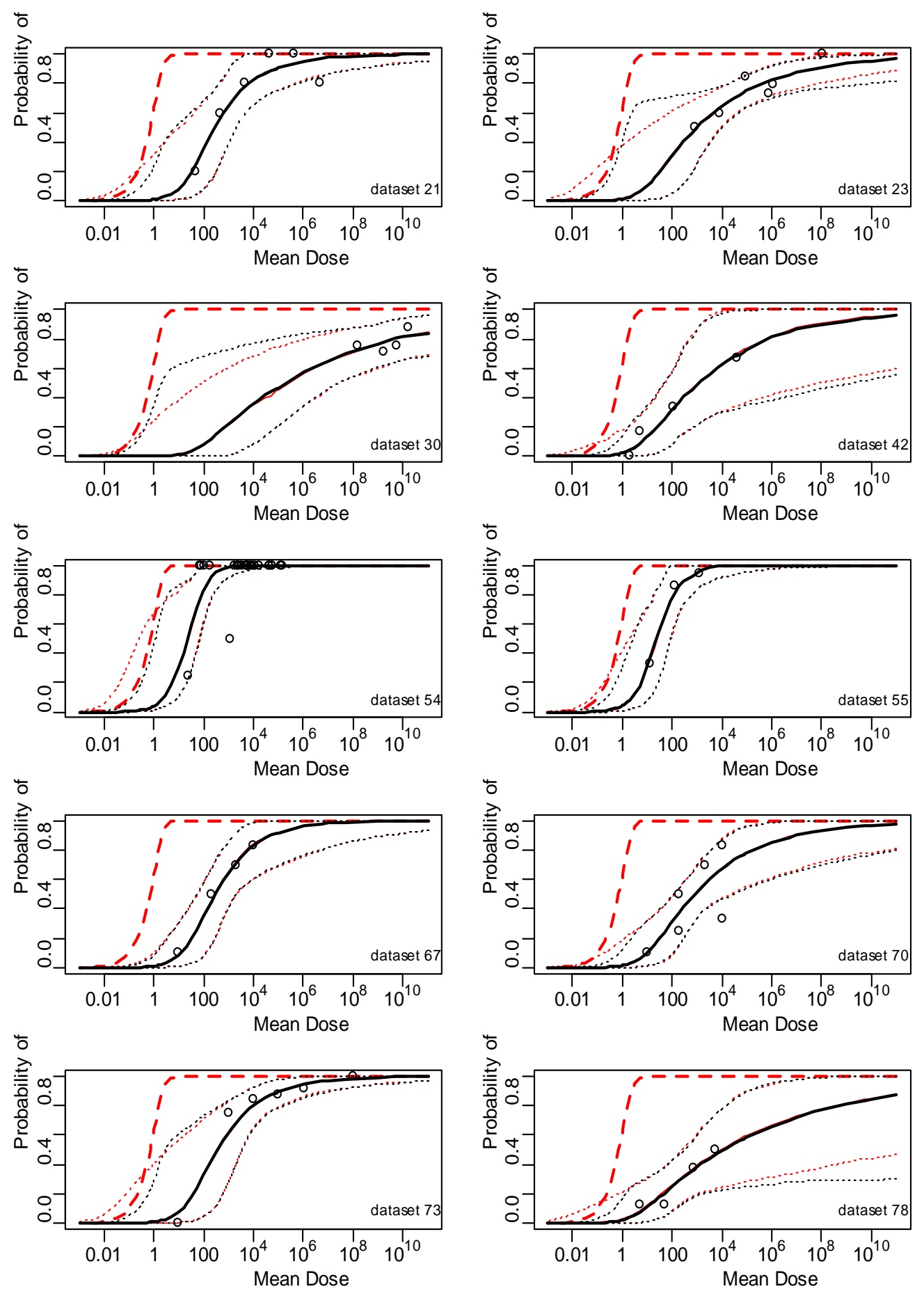

Fig. 4. Validation of the rule-of-thumb by taking into account the uncertainty around the expected doseresponse curve. Circle dots represent the fractions of the infected host subjects at different mean dose levels. The exact beta-Poisson curves are represented by bold black solid lines; the approximate betaPoisson curves are represented by red solid lines; the 95\% confidence bands are represented by doted 
lines. The bold red dashed lines represent the maximum risk dose-response curve. The horizontal axis measures the mean dose (on the natural log scale) ranged from $10^{-3}(0.001)$ to $10^{11}(100,000,000,000)$.

By examining every plot in Fig. 4, the most obvious feature is that, with no exceptions, the dose-response curves are very closely aligned (i.e., the red solid lines for the approximate models are almost completely superimposed by the bold black solid lines) as we expect, despite the different deviation patterns in the confidence bands.

Among different possible influential factors which may have caused the poor confidence bands, the mean dose level coverage range and the number of the participation subjects in each exposure group (i.e., $\mathbf{N}=$ $\left.\left\{N_{1}, N_{2}, \ldots, N_{m}\right\}\right)$ are likely two important ones worth examining closely. Consider the plot related to dataset 30 against the plot related to dataset 67 in Fig. 4. Each dataset contains four data points with parameters $\hat{\alpha}=0.086$ and $\hat{\beta}=57.4$ for dataset 30 , and $\hat{\alpha}=0.277$ and $\hat{\beta}=21.1$ for dataset 67 (Table II). From these plots it is a surprise to notice that the dataset 30 model behaves much poorly than dataset 67 model in that the upper bound curves of the confidence band deviate badly between the approximate and the exact models for dataset 30 while it is almost a perfect match for dataset 67 . We attribute the primary reason for the poor performance by dataset 30 model to the fact that its mean dose level coverage range is extremely biased towards the higher end of the horizontal axis (e.g., no data points for $d<10^{8}$, Table IV). In contrast, the near perfect match in confidence bands for dataset 67 model is due to its good coverage of the mean dose levels across the horizontal axis range. A similar conclusion may be drawn if we apply this logical reasoning to examine plots related to datasets $23,70,73$, and 78 . Each of these four datasets contains four or six data points. Those datasets with better coverage of the mean dose levels have better matching of the confidence bands (e.g., dataset 70).

By examining the deviation pattern displayed in the plot related to dataset 54 (a dataset with 24 data points, Table IV), a different influence factor is identified. It is noted that most of the data points in dataset 54 has a very small number of participated subjects in the exposure groups, e.g., $N_{i}=2$. This 
implies that the observed response $\pi_{i}^{o}=y_{i} / N_{i}$ can only be one of the three possible outcomes: $\{0 / 2,1 / 2$, $2 / 2\}$, while theoretically $\pi_{i}^{o}$ should allow to take any value between zero and one. This $N_{i}=2$ effect is clearly shown in the plot related to dataset 54 in Fig. 4 - all data points, except two, fall on the horizontal $P_{I}(d)=1$ line. As a consequence, the upper bond curve of the dataset 54 model's confidence band not only deviates from the exact model badly also go far beyond the maximum risk dose-response curve. In contrast, those models with data points well spread over the vertical axis have shown a much better pattern in matching the confidence band. For datasets 21, 42, and 55, the confidence band deviation or the violation of the maximum risk dose-response boundary also occur but to a lesser extent.

The above analysis results have the implications for us to make the following suggestion (with regard to a valid application of Equation (1) by taking into account the uncertainty): the quality of the dose-response data should not be ignored in assessing the validity of Equation (1) for dose-response analysis. Very biased data coverage, either on horizontal axis (the mean dose level coverage) or on vertical axis (the observed infected subject fractions coverage) will cause severe information loss (or is an indication for lack of information), hence unreliable confidence bands outside the data range are expected for both the approximate and the exact model. Therefore, as long as it is explicitly stated that the $95 \%$ confidence band is constructed based on Equation (1), use it anyway for QMRA because the approximation remains reasonably good within the data coverage range as shown in Fig. 4 and neither confidence bands are the reliable estimates of the true risk upper limit outside the data range. Over the mean dose range where the upper bound curve of the approximate model confidence band goes beyond the possible risk limit, the maximum risk dose-response curve should be used instead as the upper bound.

\section{CONCLUSIONS}

It is not unusual that researchers or practitioners are deterred from using the exact beta-Poisson doseresponse model in QMRA practice. This is due to the mathematical complexity in its model specification and the difficulty in parameter estimation (e.g., when conducting QMRA using the Microsoft Excel as an analysis platform). The current guidelines ( $\alpha \ll \beta$ and $\beta \gg 1$ ) for a valid application of the widely 
accepted, user-friendly approximate beta-Poisson model formula, $P_{I}(d)=1-\left(1+\frac{d}{\beta}\right)^{-\alpha}$, on the other hand, are not specific enough to apply. With the aim of providing better guidelines for the valid application of the approximate beta-Poisson model in QMRA, this study has proposed a useful rule-ofthumb, $\hat{\beta}>(22 \hat{\alpha})^{0.50}$ for $0.02<\hat{\alpha}<2$. This rule-of-thumb can be used as a sufficient condition to ensure that the approximate model (Equation 1) is in fact an accurate approximation of the exact betaPoisson model (Equation 4) in a vast range of practical situations. This rule is based on solid theoretical grounds and essentially, has relaxed the constraint of $\beta \gg 1$ that is required in current guidelines. The performance of this rule-of-thumb was validated by applying it to the 85 completed beta-Poisson models from the QMRA wiki, which were based on experimental dose-response datasets reported in the literature. The validation results showed that the proposed rule-of-thumb ensured a near perfect match in doseresponse curves between the approximate and the exact models for all the 68 cases which were identified as valid applications (i.e., the rule-of-thumb condition was satisfied). By taking parameter estimation uncertainty into account, the validation results further indicated that, given a good match in the doseresponse curve, any substantial deviations in the 95\% bootstrap confidence bands signified a need for better dose-response data to ensure a reliable QMRA outcome. Of the 85 beta-Poisson models examined here, there were 17 cases of invalid application of the approximate model identified by the rule-of-thumb. The majority of these invalid cases (13 out of 17) showed clear (i.e., visually identifiable) discrepancies in matching the dose-response curves and, therefore, they represented cases where the exact beta-Poisson model should have been applied. For the four remaining invalid cases (in which the approximation performances were much better than the above mentioned 13 cases), it was recommended that researchers should use their discretion to decide if the approximate model should be considered as a valid application on an ad hoc basis. The research findings reported in this paper provide improved guidance that will ensure the valid application of the approximate beta-Poisson formula. In turn, use of the proposed ruleof-thumb should instil more confidence in the applications of the approximate beta-Poisson dose-response model in microbial health risk assessment practice. 


\section{ACKNOWLEDGMENTS}

This article is a part of the research results of the pond project funded by Department of Science, Information Technology and Innovation (DSITI) of Queensland government, Australia. The authors would like to thank Professor Charles Haas for his valuable comments and advice on the manuscript. Author KM acknowledges research support from the QUT Institute for Future Environments and the ARC Centre of Excellence for Mathematical and Statistical Frontiers.

\section{REFERENCES}

1. Haas CN, Rose JB, Gerba CP. Quantitative Microbial Risk Assessment. second ed. New York: John Wiley \& Sons, INC.; 2014.

2. Rose J, Haas C, Gurian P, Mitchell J, Weir M. QMRA Wiki Center for Advancing Microbial Risk Assessment; [updated 23 January, 2015; cited 2016 February]. Quantitative Microbial Risk Assessment (QMRA) Wiki]. Available from: http://qmrawiki.canr.msu.edu/index.php/Quantitative_Microbial_Risk_Assessment_\%28QMRA\%29 -Wiki.

3. Mara D, Sleigh P, Blumenthal U, Carr R. Health risks in wastewater irrigation: comparing estimates from quantitative microbial risk analyses and epidemiological studies. Journal of water and health. 2007;5(1):39-50. doi: 10.2166/wh.2006.055.

4. Karavarsamis N, Hamilton A. Estimators of annual probability of infection for quantitative microbial risk assessment. Journal of water and health. 2010;8(2):365-73.

5. WHO. Guidelines for Drinking-water Quality. In: Organization WH, editor. Geneva: World Health Organization; 2008.

6. the Interagency MRA Guideline Workgroup. Microbial Risk Assessment Guideline, Pathogenic Microorganisms with Focus on Food and Water. U.S. Environment Protection Agency (EPA) and U.S. Department of Agriculture/Food Safety and Inspection Service (USDA/FSIS); 2012.

7. US EPA. Guidelines for Water Reuse. In: Agency USEP, editor. Washington: U.S. Environmental Protection Agency; 2004.

8. NRMMC, EPHC, AHMC. AUSTRALIAN GUIDELINES FOR WATER RECYCLING: MANAGING HEALTH AND ENVIRONMENTAL RISKS (PHASE1). Canberra, Australia: the Environment Protection and Heritage Council, the Natural Resource Management Ministerial Council and the Australian Health Ministers’ Conference; 2006.

9. WHO. WHO guidelines for the safe use of wastewater, excreta and greywater. Volume 2. Geneva, Swizerland: World Health Organization; 2006. 
10. NHMRC AG. Guidelines for Managing Risks in Recreational Water. In: National Health and Medical Research Council AG, editor. Canberra: Australian Government; 2008.

11. Organization WH. Food safety risk analysis: A guide for national food safety authorities. 2006.

12. Teunis P, Takumi K, Shinagawa K. Dose response for infection by Escherichia coli O157: H7 from outbreak data. Risk Analysis. 2004;24(2):401-7.

13. Teunis P, van der Heijden OG, van der Giessen JWB, Havelaar A. The dose-response relation in human volunteers for gastro-intestinal pathogens. The netherlands: National Institute of Public Health and the Environment (RIVM), 1996 Contract No.: Report nr. 284550002.

14. Xie G, Roiko A, Stratton H, Lemckert C, Dunn PK, Mengersen K. A Generalized QMRA BetaPoisson Dose-Response Model. Risk Analysis. 2016. doi: 10.1111/risa.12561.

15. Teunis P, Havelaar A. The Beta Poisson Dose-Response Model Is Not a Single-Hit Model. Risk Analysis. 2000;20(4):513-20.

16. Furumoto WA, Mickey R. A mathematical model for the infectivity-dilution curve of tobacco mosaic virus: Theoretical considerations. Virology. 1967;32(2):216-23. doi: http://dx.doi.org/10.1016/0042-6822(67)90271-1.

17. Kreyszig E. Advanced engineering mathematics. 10th edition ed: John Wiley \& Sons; 2011.

18. Haas CN. Conditional dose-response relationships for microorganisms: Development and application. Risk analysis. 2002;22(3):455-63.

19. Muller KE. Computing the confluent hypergeometric function, M (a, b, x). Numerische Mathematik. 2001;90(1):179-96.

20. Butler RW, Wood ATA. Laplace Approximations for Hypergeometric Functions with Matrix Argument. The Annals of Statistics. 2002;30(4):1155-77.

21. Krishnamoorthy K. Handbook of statistical distributions with applications: CRC Press; 2006.

22. Schmidt PJ, Pintar KD, Fazil AM, Topp E. Harnessing the Theoretical Foundations of the Exponential and Beta-Poisson Dose-Response Models to Quantify Parameter Uncertainty Using Markov Chain Monte Carlo. Risk Analysis. 2013;33(9):1677-93.

23. McCullagh P, Nelder J. Generalized linear models. London: Chapman and Hall; 1989.

24. Morgan BJ. Analysis of quantal response data: CRC Press; 1992.

25. R Development Core Team. R: A Language and Environment for Statistical Computing Vienna, Austria: R Foundation for Statistical Computing; 2014. Available from: http://www.R-project.org.

26. Hankin RK. Package 'gsl'. 2013.

27. Crawley MJ. The R book. second edition ed: John Wiley \& Sons; 2013.

28. Haas CN, Rose JB, Gerba CP. Quantitative Microbial Risk Assessment. first ed. New York: John Wiley \& Sons, INC.; 1999. 
29. Efron B, Tibshirani RJ. An introduction to the bootstrap: CRC press; 1993.

\section{APPENDIX: DATASETS AND CURVE FITTING DETAILS}

Table III. The list of the experimental dose-response datasets from QMRA literature which have

been fitted with the approximate beta-Poisson models: these datasets are described and presented, and the model fitting results are provided in the QMRA Wiki ${ }^{(2)}$.

\begin{tabular}{|c|c|c|}
\hline Dataset & Description of the dataset & $\begin{array}{l}\text { Organism } \\
\text { group }\end{array}$ \\
\hline 1 & Mycobacterium avium experiment 263 dose response data & Bacteria \\
\hline 2 & $\begin{array}{l}\text { Rickettsia rickettsi (Rocky Mountain spotted Fever) experiment } 244 \text { Rhesus } \\
\text { monkey data }\end{array}$ & Bacteria \\
\hline 3 & $\begin{array}{l}\text { Salmonella nontyphoid experiment } 246 \text { mice/Salmonella strain } 216 \text { and } 219 \\
\text { data }\end{array}$ & Bacteria \\
\hline 4 & Rhinovirus experiment 65 human/type 39 strain SF 299 model data & Viruses \\
\hline 5 & Rhinovirus experiment 64 human/type 14 strain SF 765model data & Viruses \\
\hline 6 & Rhinovirus experiment 310 data & Viruses \\
\hline 7 & Rhinovirus experiment 311 data & Viruses \\
\hline 8 & Rhinovirus experiment 312 data & Viruses \\
\hline 9 & Rhinovirus experiment 313 data & Viruses \\
\hline 10 & Rhinovirus experiment 312,313 pooled data & Viruses \\
\hline 11 & Rotavirus experiment 70, model data for rotavirus (CJN strain) in the human & Viruses \\
\hline 12 & Rotavirus experiment 71 , model data for rotavirus (CJN strain) in the human & Viruses \\
\hline 13 & Rotavirus experiment 125 , model data for rotavirus (CJN strain) in the human & Viruses \\
\hline 14 & $\begin{array}{l}\text { Cryptosporidium parvum and Cryptosporidium hominis experiment } 181 \\
\text { TU502 data }\end{array}$ & Protozoa \\
\hline 15 & $\begin{array}{l}\text { Cryptosporidium parvum and Cryptosporidium hominis experiment 183, } \\
\text { Moredunn isolate data }\end{array}$ & Protozoa \\
\hline 16 & Cryptosporidium parvum and Cryptosporidium hominis experiment 141 data & Protozoa \\
\hline 17 & Endamoeba coli experiment 45 humans model data & Protozoa \\
\hline 18 & Bacillus anthracis guinea pig/Vollum Strain model data (Experiment 84) & Bacteria \\
\hline 19 & $\begin{array}{l}\text { Burkholderia pseudomallei (B. pseudomallei) experiment } 18 \text { and } 23 \text { pooled } \\
\text { data of C57BL/6 mice and diabetic rat }\end{array}$ & Bacteria \\
\hline 20 & $\begin{array}{l}\text { Burkholderia pseudomallei (B. pseudomallei) experiment } 21 \text { and } 23 \text { pooled } \\
\text { data of guinea pig and diabetic rat }\end{array}$ & Bacteria \\
\hline 21 & $\begin{array}{l}\text { Burkholderia pseudomallei (B. pseudomallei) experiment } 21 \text { guinea pigs } \\
\text { W294 strain data }\end{array}$ & Bacteria \\
\hline 22 & $\begin{array}{l}\text { Burkholderia pseudomallei (B. pseudomallei) experiment } 23 \text { data of diabetic } \\
\text { rat and 316c strain }\end{array}$ & Bacteria \\
\hline 23 & $\begin{array}{l}\text { Campylobacter jejuni and Campylobacter coli experiment } 106 \text { Strain A3249 } \\
\text { model data }\end{array}$ & Bacteria \\
\hline 24 & $\begin{array}{l}\text { Campylobacter jejuni and Campylobacter coli experiment } 185 \text { T2 Strain for } \\
\text { serotype PEN } 2 \text { model data }\end{array}$ & Bacteria \\
\hline
\end{tabular}



serotype PEN 3 data model data the human model data paper as “type 55, B5")) in the human model data paper as "E. coli 111, B4")) in the human model data (ST)) in the human model data

Pathogenic Escherichia coli experiment 38,39,40,42,99,144 Escherichia coli disease (ETEC, EPEC, EIEC) in the human model data disease in the human model data

EPEC disease in the human model data Pathogenic Escherichia coli experiment 38,42,99,165 Escherichia coli ETEC disease, unbuffered, in the human model data

Bacteria

Bacteria

Bacteria

Bacteria

Bacteria

Bacteria

Bacteria

Bacteria

Bacteria

Bacteria

Bacteria

Bacteria

Bacteria

Bacteria

Bacteria

Bacteria

Bacteria

Bacteria

Bacteria

Bacteria

Bacteria

Bacteria

Bacteria

Bacteria

Bacteria

Bacteria

Bacteria 
day2-21/Pseudomonas aeruginosa

$52 \quad$ Pseudomonas aeruginosa (bacterimia) experiment 283,284 pooled

Bacteria mice(10day old, day1-21 pooled/Pseudomonas aeruginosa

53 Rickettsia rickettsi (Rocky Mountain spotted Fever) pooled data (experiment no. 300 and 301)

54 Rickettsia rickettsi (Rocky Mountain spotted Fever) experiment 300 Rhesus monkey data

55 Rickettsia rickettsi (Rocky Mountain spotted Fever) experiment 301 human data

Salmonella Typhi experiment 79 and 80 pooled model data for S. Typhi (Quailes) in humans

Salmonella Typhi experiment 79 model data for S. Typhi (Quailes) in humans Salmonella Typhi experiment 80 model data for $S$. Typhi (Quailes) in humans Salmonella anatum experiment 232, 233 pooled human/ Salmonella anatum strain I \& II pooled data

Salmonella anatum experiment 232 human/ Salmonella anatum strain I \& II pooled data

Salmonella anatum experiment 233 human/ Salmonella anatum strain I \& II pooled data

Salmonella meleagridis experiment 238 human / S. meleagridis strain I data

Salmonella meleagridis experiment 240 human / S. meleagridis strain III data

Salmonella nontyphoid experiment 247 mice/Salmonella strain 533 data

Salmonella nontyphoid experiment 248 mice/Salmonella strain 533 data

Shigella (Shigellosis) experiment 83 model data for Shigella flexneri (2a, strain 2457T) in the human

Shigella (Shigellosis) experiment 81 model data for Shigella dysenteriae (M 131) in the human strain 2457T) in the human

Bacteria

Bacteria

Bacteria

Bacteria

Bacteria

Bacteria

Bacteria

Bacteria

Bacteria

Bacteria

Bacteria

Bacteria

Bacteria

Bacteria

Bacteria

Bacteria

Bacteria

Bacteria

Bacteria

Bacteria

Bacteria

Bacteria

Bacteria

Viruses

Viruses

Echovirus experiment 112 humans/ echovirus-12 strain

Viruses

Influenza experiment 257,258 pooled dose response data

Viruses

Influenza experiment 257 human/H1N1 A/California/10/78 attenuated strain Viruses model data strain model data 
85 PrP prions (Scrapie, BSE, CJD) experiment 250 hamsters/scrapie strain 263K Prions model data

Table IV. Dose-response experiment datasets cited from QMRA Wiki ${ }^{(2)}$ for the purposes of

\section{producing Figure 4 and the calculation of $\mathrm{N}_{50}$ for the dataset 11 case. $*$}

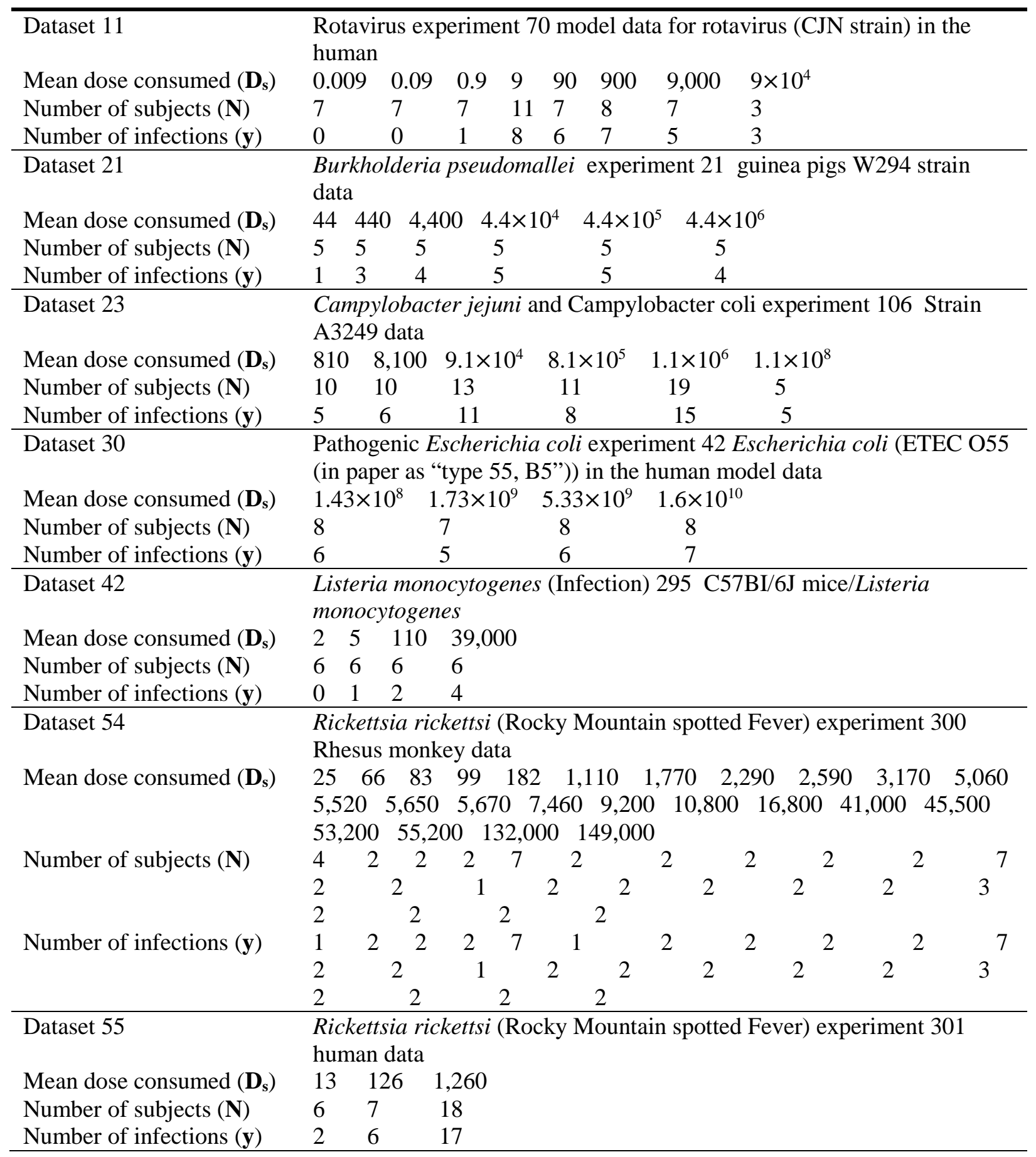




\begin{tabular}{|c|c|c|c|c|c|}
\hline Dataset 67 & \multicolumn{5}{|c|}{$\begin{array}{l}\text { Shigella (Shigellosis) experiment } 81 \text { model data for Shigella dysenteriae } \\
\text { (M 131) in the human }\end{array}$} \\
\hline Mean dose consumed $\left(\mathbf{D}_{\mathbf{s}}\right)$ & 10 & 200 & 2,00 & $0 \quad 10^{4}$ & \\
\hline Number of subjects $(\mathbf{N})$ & 10 & 4 & 10 & 6 & \\
\hline Number of infections (y) & 1 & 2 & 7 & 5 & \\
\hline Dataset 70 & \multicolumn{5}{|c|}{$\begin{array}{l}\text { Shigella (Shigellosis) experiment 81, } 215 \text { model data for Shigella } \\
\text { dysenteriae in the human }\end{array}$} \\
\hline Mean dose consumed $\left(\mathbf{D}_{\mathbf{s}}\right)$ & 10 & 200 & 200 & $2,000 \quad 10^{4}$ & $10^{4}$ \\
\hline Number of subjects $(\mathbf{N})$ & 10 & 4 & 4 & 10 & 6 \\
\hline Number of infections $(\mathbf{y})$ & 1 & 2 & 1 & 5 & 2 \\
\hline Dataset 73 & \multicolumn{5}{|c|}{ Vibrio cholerae (Cholera) experiment 249 human Inaba Strain 569B } \\
\hline Mean dose consumed $\left(\mathbf{D}_{\mathrm{s}}\right)$ & 10 & 1,000 & $10^{4}$ & $10^{5} 10^{6}$ & $10^{8}$ \\
\hline Number of subjects $(\mathbf{N})$ & 2 & 4 & 13 & 23 & 2 \\
\hline Number of infections $(\mathbf{y})$ & 0 & 3 & 11 & $\begin{array}{ll}7 & 21 \\
\end{array}$ & 2 \\
\hline Dataset 78 & \multicolumn{5}{|c|}{ Lassa virus experiment 15 guinea pig/ Josiah strain model data } \\
\hline Mean dose consumed $\left(\mathbf{D}_{\mathbf{s}}\right)$ & 5 & $48 \quad 7:$ & 245 , & \multicolumn{2}{|c|}{5,370} \\
\hline Number of subjects $(\mathbf{N})$ & 8 & 8 & & \multicolumn{2}{|c|}{8} \\
\hline Number of infections $(y)$ & 1 & 3 & & \multicolumn{2}{|c|}{4} \\
\hline
\end{tabular}

\section{Curve fitting details}

For approximation of the $\operatorname{Pr}(0<r<1 \mid \alpha, \beta)=0.99$ probability contour line through curve fitting, the best-fit power function curve $(\alpha<2)$ and the best-fit straight line $(2 \leq \alpha<20)$ are obtained using the least-squares estimation method ${ }^{(27)}$ implemented with R function 'optim'(27). The fitted power function is in the form of $\beta=(\mathrm{A} \alpha)^{\mathrm{B}}$ and the linear (i.e., straight line) function is in the form of $\beta=\mathrm{A} \alpha+\mathrm{B}$ where $\mathrm{A}$ and B are parameters to be estimated. It is a two-step process to obtain the parameter estimates. Step 1 is to obtain the paired $\alpha$ and $\beta$ values in which $\alpha$ values are first arbitrarily determined over the range $[0.001,20]$ as shown in Table $\mathrm{V}$ and then the corresponding $\beta$ values are determined by trial and error using the built-in $\mathrm{R}$ function 'pgamma'(27) such that $\operatorname{pgamma}(1, \alpha, \beta) \geq 0.99$. The paired $\alpha$ and $\beta$ values obtained in such a way accurately characterize the 0.99 probability contour line; Step 2 is to use $\mathrm{R}$ function 'optim' to find the best-fit least-squares estimates for A and B by matching the paired $\alpha$ and $\beta$ values obtained in Step 1, i.e., given the same $\alpha$ values, the optimal parameter estimates A and B minimise the differences between the $\beta$ values in Table $\mathrm{V}$ and the $\beta$ values produced from the fitted 
power function $\beta=(\mathrm{A} \alpha)^{\mathrm{B}}$ (for $\left.\alpha \leq 2\right)$ or the linear function $\beta=\mathrm{A} \alpha+\mathrm{B}$ (for $2 \leq \alpha \leq 20$ ). The paired $\alpha$ and $\beta$ values in the top two rows of Table $\mathrm{V}$ are used for fitting the power function $\beta=(\mathrm{A} \alpha)^{\mathrm{B}}$; the paired $\alpha$ and $\beta$ values in the bottom two rows are used for fitting the linear function $\beta=\mathrm{A} \alpha+\mathrm{B}$.

The optimal parameter estimates have been found as A = 22 (rounded to 2 significant figures (2 sf)) and B $=0.50(2 \mathrm{sf})$ for the power function, and $\mathrm{A}=1.4(2 \mathrm{sf})$ and $\mathrm{B}=4.4(2 \mathrm{sf})$ for the linear function, respectively.

Table V. Paired $\alpha$ and $\boldsymbol{\beta}$ values used for characterizing the probability contour line of $\operatorname{Pr}(0<r<$ $1 \mid \alpha, \beta)=0.99$

\begin{tabular}{|c|c|c|c|c|c|c|c|c|c|c|c|c|c|c|c|c|}
\hline$\alpha$ & 0.001 & & 0.005 & 0.01 & 0.02 & 0.03 & 0.04 & 0.07 & 0.1 & 0.2 & 0.4 & 0.6 & 0.8 & 1 & 1.5 & 2 \\
\hline$\beta$ & 0.000 & & 0.083 & 0.27 & 0.57 & 0.79 & 0.96 & 1.33 & 1.60 & 2.22 & 3.02 & 3.63 & 4.15 & 4.63 & 5.70 & 6.65 \\
\hline$\alpha$ & 2 & 4 & 8 & 12 & 15 & 20 & & & & & & & & & & \\
\hline$\beta$ & 6.65 & 10.1 & 16.0 & 21.5 & 25.5 & 31.9 & & & & & & & & & & \\
\hline
\end{tabular}

